\title{
Thrombocytopenia in a cohort of primary and secondary antiphospholipid syndrome patients: Relation to clinical, laboratory manifestations and damage index
}

\author{
Sherif Gamal(D), Samar Mohamed(D, Abdelkawy Moghazy (D) \\ Department of Rheumatology, Faculty of Medicine, Cairo University, Cairo, Egypt
}

\begin{abstract}
Objectives: This study aims to evaluate the prevalence of thrombocytopenia in a cohort of patients with primary and secondary antiphospholipid syndrome (APS) and to examine the relation of thrombocytopenia to the clinical, laboratory findings, and damage index for antiphospholipid syndrome (DIAPS).

Patients and methods: Between August 2018 and February 2019, a total of 168 patients (16 males, 152 females; mean age: $32.5 \pm 8.4$ years; range, 18 to 59 years) who were followed in our clinic for APS were retrospectively analyzed. Medical records of the patients were screened and clinical data, laboratory investigations, and treatments applied were recorded. The DIAPS was calculated for all patients. The patients were divided into two groups according to the presence or absence of thrombocytopenia and both groups were compared regarding clinical, laboratory findings and DIAPS. Further subgroup analysis was done for patients with primary APS.

Results: The most common clinical manifestations in our patients were obstetric manifestations ( $77.4 \%$ in pregnant women), musculoskeletal manifestations (69\%) and peripheral vascular thrombosis (54.8\%). The prevalence of thrombocytopenia in our study was $42.3 \%$, and it was significantly associated with musculoskeletal manifestations $(p=0.043)$, vascular thrombosis $(p=0.043)$, neurological manifestations $(p=0.030)$, cutaneous manifestations ( $p=0.006)$, and use of immunosuppressives $(p=0.047)$. The DIAPS was significantly higher in the thrombocytopenia group $(p=0.034)$. Further subgroup analysis of patients with primary APS revealed that neurological manifestations $(p=0.010)$ were significantly higher in the thrombocytopenia group, while the DIAPS was higher in the thrombocytopenia group, but it did not reach statistical significance ( $p=0.082)$.

Conclusion: Thrombocytopenia may be associated with a higher incidence of vascular thrombosis, neurological manifestations, musculoskeletal manifestations, use of immunosuppressive treatment, and DIAPS. In primary APS patients, thrombocytopenia may be a risk for neurological manifestations.
\end{abstract}

Keywords: Antiphospholipid syndrome, damage index, Egyptian patients, thrombocytopenia.

Antiphospholipid syndrome (APS) is an autoimmune-mediated acquired thrombophilia. ${ }^{1,2}$ It is defined as a syndrome of recurrent venous and/or arterial thromboses, pregnancy complication together with the presence of antiphospholipid antibodies (aPL), that are directed against phospholipid-binding plasma proteins. ${ }^{1-3}$
Thrombocytopenia is one of the most common non-criteria findings in APS patients. ${ }^{4}$ The estimated prevalence of thrombocytopenia in patients with APS ranged from 20 to 53\%, depending on the cut-off value used $\left(<100 \times 10^{9} / \mathrm{L}\right.$ or $\left.<150 \times 10^{9} / \mathrm{L}\right),{ }^{5}$ and the frequency of patients with primary APS (PAPS) and secondary APS

Received: July 03, 2021 Accepted: September 06, 2021 Published online: January 23, 2022

Correspondence: Sherif Gamal, MD. Egypt-Cairo-Nasr City-Emtedad Ramsis Street-4 Elgabal Elakhdar Building, 11562 Cairo, Egypt. Tel: 00201001811162 e-mail: sherif775@hotmail.com

\section{Citation:}

Gamal S, Mohamed S, Moghazy A. Thrombocytopenia in a cohort of primary and secondary antiphospholipid syndrome patients: Relation to clinical, laboratory manifestations and damage index. Arch Rheumatol 2022;37(2):252-260. 
(SAPS) in the studied cohort. ${ }^{6-8}$ Interestingly, the prevalence of thrombocytopenia may reach up to 65 to 100\% in patients with catastrophic APS (CAPS). ${ }^{8,9}$ Thus, some authors have suggested that a progressive decrease in the platelet count in APS patients may be considered a sign for progressive disease and be a risk factor for developing CAPS in the future. ${ }^{1,10}$ Furthermore, Artim-Esen et al. ${ }^{4}$ reported coexistence of thrombocytopenia and neuropsychiatric manifestations, in association with lupus anticoagulant and high titers of anticardiolipin antibodies.

Although several studies have been conducted on thrombocytopenia in APS patients to date, ${ }^{6,11-13}$ there are important issues that still need to be addressed, such as relation of thrombocytopenia to clinical associations of APS, its effect on the disease outcomes, and finally the relation of thrombocytopenia to the APS damage index (DIAPS). In the present study, we aimed to evaluate the prevalence of thrombocytopenia in patients with APS and to examine the relation of thrombocytopenia to the clinical and laboratory findings, and DIAPS.

\section{PATIENTS AND METHODS}

This single-center, retrospective study was conducted at Cairo University Hospital, Faculty of Medicine, Department of Rheumatology, between August 2018 and February 2019. A total of 168 patients (16 males, 152 females; mean age: $32.5 \pm 8.4$ years; range, 18 to 59 years) who were followed in our clinic and fulfilled the update of Sapporo Classification Criteria for definite APS ${ }^{14}$ were included. The medical records were revised for demographic data, clinical features of the disease, immune profile, routine laboratory investigations, and treatments applied. In addition, the DIAPS ${ }^{15}$ was calculated for all patients. The patients were divided into two groups according to the presence or absence of thrombocytopenia and both groups were compared regarding demographic data, clinical, laboratory findings, treatments, and DIAPS. Patients less than 18 years old or with disease duration of less than two years were excluded from the study. Thrombocytopenia was considered a clinical feature of APS in our study, when the platelet count of less than $150 \times 10^{9} / \mathrm{L}$ was found on a minimum of two occasions. ${ }^{11}$
Further subgroup analysis was done for patients with PAPS according to the presence or absence of thrombocytopenia.

\section{Statistical analysis}

Statistical analysis was performed using the SPSS for Windows version 23.0 software (IBM Corp., Armonk, NY, USA). Descriptive data were expressed in mean \pm standard deviation (SD) or median (min-max) for quantitative variables and in number and frequency for qualitative variables. Comparison between the groups was conducted using the Student t-test for normally distributed data and Mann-Whitney $U$ test for skewed data. The chi-square test was used analyze qualitative variables. A $p$ value of $<0.05$ was considered statistically significant.

\section{RESULTS}

The mean disease duration was $9.3 \pm 6.1$ years, and the mean age at onset was $23.3 \pm 7.6$ years. Forty-five (26.8\%) patients had PAPS, 116 (69\%) patients had APS secondary to systemic lupus erythematosus (SLE), while seven (4.2\%) patients had APS secondary to other diseases. The most commonly reported manifestations in our patients were obstetric manifestations (77.4\% in pregnant women), musculoskeletal manifestations (69\%), and peripheral vascular thrombosis (54.8\%) (Table 1).

The laboratory features of our patients in the last visit, their treatment and DIAPS are shown in Table 2. Our patients were divided into two groups according to presence or absence of thrombocytopenia, the two groups were compared regarding demographic, clinical, laboratory, treatments, and DIAPS as shown in Table 3. Furthermore, we studied patients with PAPS to exclude any impact of associated disease, and we divided them into two groups according to the presence or absence of thrombocytopenia and both groups were compared regarding the previously mentioned items as shown in Table 4.

\section{DISCUSSION}

The estimated prevalence of thrombocytopenia in APS in the literature ranges between 
Table 1. Demographic data, clinical manifestations, and immune profile of APS patients $(n=168)$

\begin{tabular}{|c|c|c|c|c|c|c|}
\hline & $\mathrm{n}$ & $\%$ & Mean \pm SD & Range & Median & IQR \\
\hline Age (year) & & & $32.5 \pm 8.5$ & $18-59$ & 32 & $26-37$ \\
\hline $\begin{array}{l}\text { Sex } \\
\text { Male } \\
\text { Female }\end{array}$ & $\begin{array}{r}9.5 \\
90.5\end{array}$ & $\begin{array}{c}16 \\
152\end{array}$ & & & & \\
\hline Age at onset (year) & & & $23.3 \pm 7.6$ & $11-52$ & 22 & $18-25$ \\
\hline Disease duration (year) & & & $9.3 \pm 6.1$ & $1-41$ & 8 & $4.5-13$ \\
\hline Time till diagnosis (year) & & & $3 \pm 3.8$ & $0-25$ & 2 & $0.3-4.5$ \\
\hline Obstetric manifestations related to APS & 103 & 77.4 & & & & \\
\hline Musculoskeletal manifestations & 116 & 69 & & & & \\
\hline Hematological manifestations (anemia-leukopenia-thrombocytopenia) & 81 & 48.2 & & & & \\
\hline Hemolytic anemia & 24 & 14.3 & & & & \\
\hline Thrombocytopenia at onset & 51 & 30.4 & & & & \\
\hline Thrombocytopenia throughout disease & 71 & 42.3 & & & & \\
\hline Constitutional manifestations & 86 & 51.2 & & & & \\
\hline Peripheral vascular thrombosis & 92 & 54.8 & & & & \\
\hline Venous thrombosis & 78 & 46.4 & & & & \\
\hline Arterial thrombosis & 24 & 14.3 & & & & \\
\hline $\begin{array}{l}\text { Neurological manifestations } \\
\text { (Seizures, stroke, TIA, psychosis) }\end{array}$ & 69 & 41.1 & & & & \\
\hline $\begin{array}{l}\text { Cardiovascular manifestations } \\
\text { (valve disease, pericardial effusion, coronary vascular diseas) }\end{array}$ & 68 & 40.5 & & & & \\
\hline $\begin{array}{l}\text { Cutaneous manifestations } \\
\text { (Thrombophlebitis, digital gangrene, cutaneous ulcers, } \\
\text { livido reticularis) }\end{array}$ & 56 & 33.3 & & & & \\
\hline $\begin{array}{l}\text { Pulmonary manifestations } \\
\text { (Pulmonary hypertension, embolism, insufficiency) }\end{array}$ & 34 & 20.2 & & & & \\
\hline $\begin{array}{l}\text { Renal manifestations } \\
\text { (Nephritis, thrombotic microangiopathy, renal failure, } \\
\text { renal artery/vein thrombosis) }\end{array}$ & 61 & 36.3 & & & & \\
\hline $\begin{array}{l}\text { Ophthalmological manifestations } \\
\text { (Retinal vasculitis, retinal thrombosis) }\end{array}$ & 11 & 6.5 & & & & \\
\hline $\begin{array}{l}\text { Hepatic and GI manifestations } \\
\text { (Budd chiari and mesenteric thrombosis) }\end{array}$ & 6 & 3.6 & & & & \\
\hline $\begin{array}{l}\text { Associated disease } \\
\text { Hypertension } \\
\text { Diabetes } \\
\text { Others }\end{array}$ & $\begin{array}{l}56 \\
43 \\
11 \\
11\end{array}$ & $\begin{array}{c}33.3 \\
25.6 \\
6.5 \\
6.5\end{array}$ & & & & \\
\hline Antinuclear antibody & 127 & 75.6 & & & & \\
\hline Anti-double-stranded deoxyribonucleic acid & 59 & 35.1 & & & & \\
\hline Lupus anticoagulant & 97 & 57.7 & & & & \\
\hline ACL IgG & 86 & 51.2 & & & & \\
\hline ACL IgM & 54 & 32.1 & & & & \\
\hline Anti-B2 glycoprotein IgG & 41 & 24.4 & & & & \\
\hline Anti-B2 glycoprotein IgM & 26 & 15.5 & & & & \\
\hline
\end{tabular}

APS: Antiphospholipid syndrome; SD: Standard deviation; IQR: Interquartile range; TIA:Transient ischemic attack; GI: Gastrointestinal; ACL: Anti-cardiolipin antibodies; Ig: Immunoglobulin. 
Table 2. Laboratory features, treatment and DIAPS

\begin{tabular}{|c|c|c|c|c|c|c|}
\hline & $\mathrm{n}$ & $\%$ & Mean \pm SD & Range & Median & IQR \\
\hline Hemoglobin & & & $11 \pm 1.8$ & $6.7-18.6$ & 11 & $10-12.1$ \\
\hline Total leucocytic count & & & $7.6 \pm 6.3$ & $0.5-66$ & 7 & $5-8.3$ \\
\hline Erythrocyte sedimentation rate & & & $41.2 \pm 35.5$ & $2-337$ & 32 & $20-54.5$ \\
\hline Alanin aminotransferaz & & & $25.4 \pm 35.4$ & $3-380$ & 19 & $14.5-27.5$ \\
\hline Creatinine & & & $0.9 \pm 0.9$ & $0.3-8$ & 0.7 & $0.5-0.9$ \\
\hline Anticoagulation & 119 & 70.8 & & & & \\
\hline Antiplatelet & 150 & 89.3 & & & & \\
\hline Corticosteroids & 141 & 83.9 & & & & \\
\hline Antimalarial & 139 & 82.7 & & & & \\
\hline Immunosuppressive treatment & 124 & 73.8 & & & & \\
\hline DIAPS & & & $2.2 \pm 2.1$ & $0-9$ & 2 & $0-4$ \\
\hline
\end{tabular}

20 and $50 \%, 4,6$ which is consistent with our results (42.3\%). The similarity in the frequency of thrombocytopenia between males and females in the current study (43.75\% and $42.1 \%)$ is in accordance with that reported in previous studies. $^{6,7}$

Regarding obstetric manifestations, we could not detect significant associations between thrombocytopenia and obstetric morbidity in our patients. On the other hand, our results showed a high rate of overall neurological manifestations in thrombocytopenia group compared to non-thrombocytopenia group $(50.7 \%$ vs. $34 \%)$ with a statistically significant difference $(p=0.03)$. We also found a strong association between thrombocytopenia and musculoskeletal manifestations $(77.5 \%$ vs. $62.9 \%)$ $(p=0.043)$. Similarly, Krause et al. ${ }^{6}$ reported that obstetric complications were similar in patients with and without thrombocytopenia and that the presence of thrombocytopenia might be a risk factor for neurological and articular manifestations. Also, Artim-Esen et al. ${ }^{4}$ reported that thrombocytopenia represents a risk factor for thrombosis and neurological manifestations in aPL-positive patients.

Our results showed a high rate of venous thrombosis in thrombocytopenia group compared to non-thrombocytopenia group $(50.7 \%$ vs. $43.3 \%)$; however, this was not statistically significant $(p=0.342)$. A high rate of arterial thrombosis was also found in thrombocytopenia group compared to the other, but also this was not statistically significant $(18.3 \%$ vs. $11.3 \%) \quad(p=0.202)$. Also, total peripheral vascular thrombosis was higher in the positive group compared to the negative group (63.4\% vs. $48.5 \%)$, with a $\mathrm{p}$ value quite close to the significant value $(\mathrm{p}=0.055)$. Comparing the two groups regarding total thrombotic events (peripheral vascular and internal organs), thrombocytopenia group showed a higher incidence of total thrombosis $(77.5 \%$ vs. $62.9 \%)$ with a significant $p$ value of 0.043 , although the rate of thrombosis in the study conducted by Krause et al. ${ }^{6}$ was slightly higher in the thrombocytopenia group; however, it did not reach statistical significance. On the other hand, Atsumi et al. ${ }^{16}$ reported that the presence of thrombocytopenia in patients with APS was not typically associated with hemorrhagic complications; rather, it could trigger thrombotic events. It was also found that the more severe the thrombocytopenia, the higher the possibility of future thrombosis. Also, Pontara et al. ${ }^{8}$ reported that a decrease in platelet count was associated with the development of the catastrophic form of the disease, a decrease in platelet count in highrisk APS patients should be evaluated cautiously for the disease progression to CAPs.

Furthermore, our results are in accordance with Demetrio Pablo et al., ${ }^{17}$ as they reported that aPL-positive patients who developed thrombocytopenia had a potential risk of developing thrombosis. In addition, 


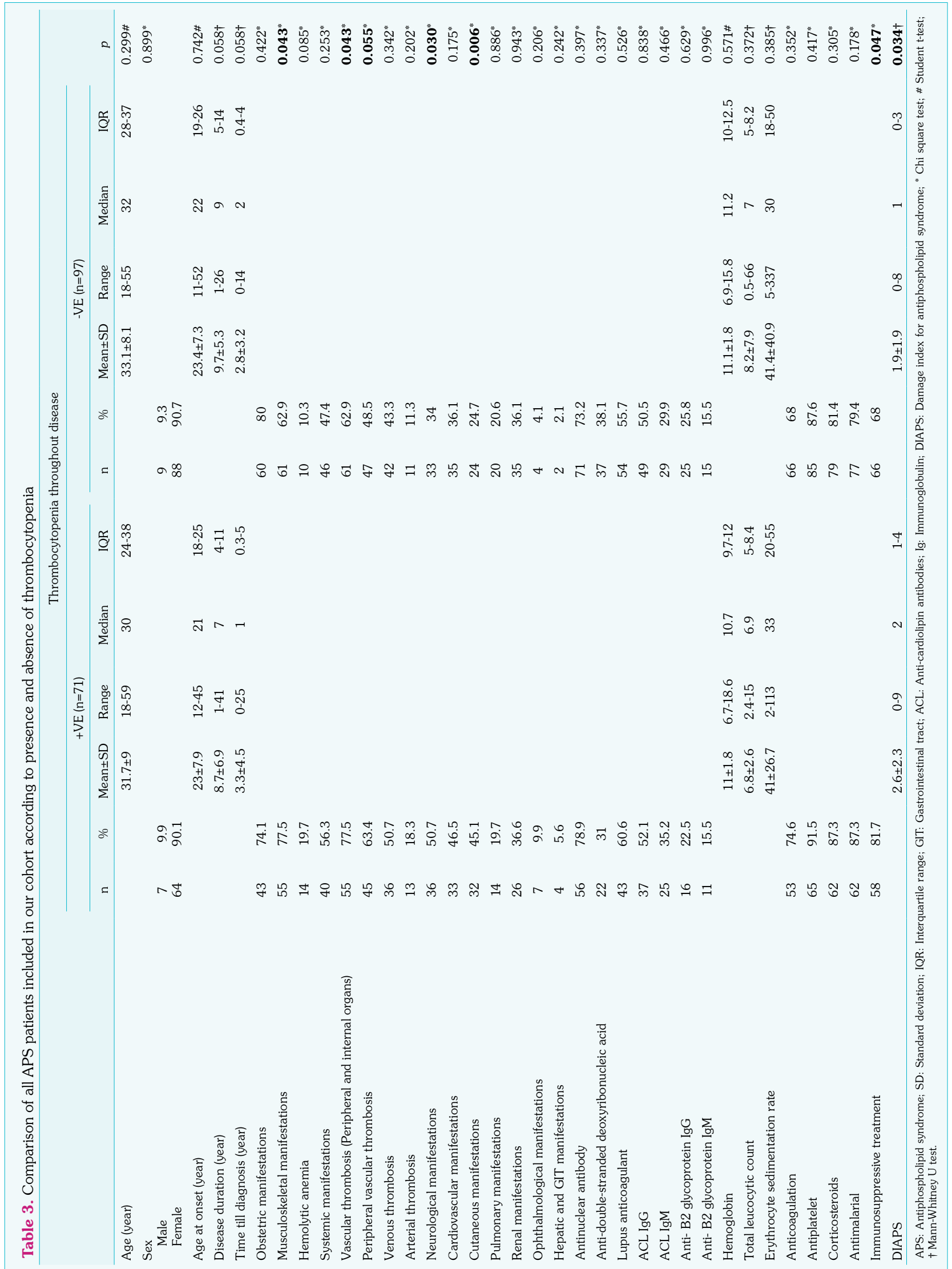




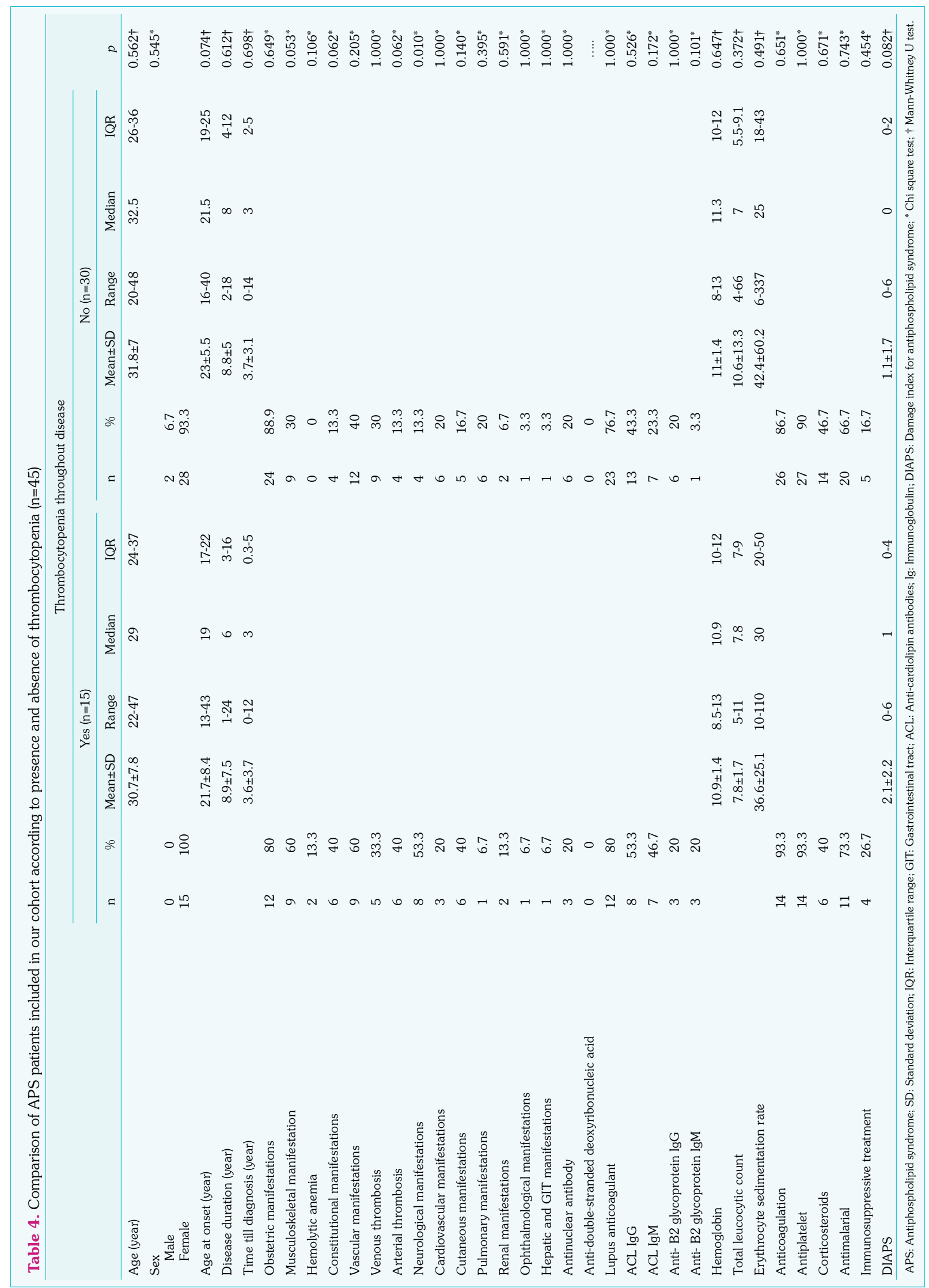


Abreu et al. ${ }^{18}$ showed that thrombocytopenia in APS was a consequence of consumption of platelets, as binding of aPL antibodies, particularly anti-beta- 2 glycoprotein antibodies, to the surface of activated platelets promoted their aggregation and thrombus formation. Finally, Proulle et al. ${ }^{19}$ showed that, among aPL-positive patients, thrombosis might develop more frequently in patients with a low platelet count, compared to those without.

Although Krause et al. ${ }^{6}$ found significant associations between thrombocytopenia and cardiac valves thickening and dysfunction, our results showed no statistically significant difference between the two groups regarding overall cardiac manifestations.

Regarding cutaneous manifestations (cutaneous ulcers, digital gangrene, livedo, thrombophlebitis), our results showed an overall increase in the rate of cutaneous manifestations in the thrombocytopenia group compared to the other group $(45.1 \%$ vs. $24.6 \%)$ with a statistically significant difference $(p=0.006)$. Similarly, Krause et al. $^{6}$ and Comellas-Kirkerup et al. ${ }^{20}$ reported significant associations between thrombocytopenia and cutaneous manifestations including livedo reticularis and skin ulcerations. Furthermore, many reports have suggested that livedo reticularis may be associated with more severe disease, as it may be associated with thrombosis, ${ }^{21}$ and also it may be associated with stroke. ${ }^{22}$ In our study, both thrombosis and stroke were significantly higher in the thrombocytopenia group and this is in line with the findings of Artim-Esen et al. ${ }^{4}$ Moreover, the association of thrombocytopenia with neurological manifestations was also confirmed in our PAPS cohort.

The significantly higher usage of immunosuppressive in the thrombocytopenia group, in our study, was expected as thrombocytopenia, particularly if severe, may require treatment with high dose glucocorticoids, immunosuppressive drugs, and rituximab along with intravenous immunoglobulin and plasma exchange. ${ }^{23-25}$

Damage index for APS was significantly higher in thrombocytopenia group $(p=0.034)$. This may be a sequence of the higher prevalence of neurological manifestations, thrombosis and cutaneous manifestations, all of which may be associated with more damage. Also, thrombocytopenia itself may be a sign of more aggressive disease and may require treatment with immunosuppressors adding to the disease burden and may affect the disease outcome. To the best of our knowledge, no previous study has compared damage accrual in APS patients with and without thrombocytopenia; however, Ruiz-Irastorza et al. ${ }^{26}$ concluded that APS associated with thrombosis might present more damage in SLE. Furthermore, Artim-Esen et al. ${ }^{4}$ reported that thrombocytopenia was a risk factor for thrombosis in aPL-positive patients. All the aforementioned data may give a reasonable explanation of the higher damage index in the thrombocytopenia group.

On analyzing PAPS patients and although DIAPS was higher in PAPS patients with thrombocytopenia $(2.1 \pm 2.2)$ compared to $(1.1 \pm 1.7)$ in those without, the difference did not reach statistical significance $(p=0.082)$. Similarly, venous thrombosis, arterial thrombosis, and total peripheral vascular thrombosis were higher in thrombocytopenia group; however, the difference did not reach statistical significance $(p=0.062)$. The lack of association of thrombocytopenia with DIAPS and arterial thrombosis, although $\mathrm{p}$ value quite close to significant in both situations, may be due to the small sample size of patients with PAPS in the current cohort. In our opinion, thrombocytopenia may represent more than an incidental laboratory finding in APS patients, and further studies may be needed to explore its role in APS pathogenesis and in damage occurrence in APS patients.

The small number of patients with PAPS included in the current study is the main limitation. We, therefore, recommend further studies on thrombocytopenia to be conducted on a large cohort of PAPS patients to avoid the impact of any associated disease as SLE on APS characteristics and DIAPs.

In conclusion, thrombocytopenia may be associated a with higher incidence of vascular thrombosis, neurological manifestations, musculoskeletal manifestations, use of immunosuppressive treatment, and DIAPS. In PAPS patients, thrombocytopenia may be a risk for neurological manifestations; however further large-scale studies are needed to confirm our findings. 
Ethics Committee Approval: The study protocol was approved by the local department committee in June 2018 and by the faculty post graduate research committee in 15 August 2018. The study was conducted in accordance with the principles of the Declaration of Helsinki.

Data Sharing Statement: The data that support the findings of this study are available from the corresponding author upon reasonable request.

Author Contributions: All authors contributed equally to the article: S.G., S.M., A.M.

Conflict of Interest: The authors declared no conflicts of interest with respect to the authorship and/or publication of this article.

Funding: The authors received no financial support for the research and/or authorship of this article.

\section{REFERENCES}

1. Linnemann B. Antiphospholipid syndrome - an update. Vasa 2018;47:451-64.

2. Omar G, Mohamed FI, Sadek HA, Mamdouh AS. Diagnostic value of anti-annexin A5 antibodies in seropositive versus seronegative antiphospholipid syndrome patients. The Egyptian Rheumatologist 2018;40:111-6.

3. Cervera R. Antiphospholipid syndrome. Thromb Res 2017;151 Suppl 1:S43-S47.

4. Artim-Esen $\mathrm{B}$, Diz-Küçükkaya $\mathrm{R}$, İnanç $\mathrm{M}$. The significance and management of thrombocytopenia in antiphospholipid syndrome. Curr Rheumatol Rep 2015;17:14.

5. Cervera R, Tektonidou MG, Espinosa G, Cabral AR, González EB, Erkan D, et al. Task Force on Catastrophic Antiphospholipid Syndrome (APS) and Non-criteria APS Manifestations (II): Thrombocytopenia and skin manifestations. Lupus 2011;20:174-81.

6. Krause I, Blank M, Fraser A, Lorber M, Stojanovich L, Rovensky J, et al. The association of thrombocytopenia with systemic manifestations in the antiphospholipid syndrome. Immunobiology 2005;210:749-54.

7. Cervera R, Piette JC, Font J, Khamashta MA, Shoenfeld Y, Camps MT, et al. Antiphospholipid syndrome: Clinical and immunologic manifestations and patterns of disease expression in a cohort of 1,000 patients. Arthritis Rheum 2002;46:1019-27.

8. Pontara E, Banzato A, Bison E, Cattini MG, Baroni G, Denas G, et al. Thrombocytopenia in high-risk patients with antiphospholipid syndrome. J Thromb Haemost 2018;16:529-32.

9. Cervera R, Rodríguez-Pintó I, Colafrancesco S, Conti F, Valesini G, Rosário C, et al. 14th International Congress on Antiphospholipid Antibodies Task Force Report on Catastrophic Antiphospholipid Syndrome. Autoimmun Rev 2014;13:699-707.
10. Provan D, Stasi R, Newland AC, Blanchette VS, Bolton-Maggs $\mathrm{P}$, Bussel $\mathrm{JB}$, et al. International consensus report on the investigation and management of primary immune thrombocytopenia. Blood 2010;115:168-86.

11. Cuadrado MJ, Mujic F, Muñoz E, Khamashta MA, Hughes GR. Thrombocytopenia in the antiphospholipid syndrome. Ann Rheum Dis 1997;56:194-6.

12. Galli M, Finazzi G, Barbui T. Thrombocytopenia in the antiphospholipid syndrome: Pathophysiology, clinical relevance and treatment. Ann Med Interne (Paris) 1996;147 Suppl 1:24-7.

13. de Groot PG. Platelets as pivot in the antiphospholipid syndrome. Blood 2014;124:475-6.

14. Miyakis S, Lockshin MD, Atsumi T, Branch DW, Brey RL, Cervera R, et al. International consensus statement on an update of the classification criteria for definite antiphospholipid syndrome (APS). J Thromb Haemost 2006;4:295-306.

15. Amigo MC, Goycochea-Robles MV, EspinosaCuervo G, Medina G, Barragán-Garfias JA, Vargas $A$, et al. Development and initial validation of a damage index (DIAPS) in patients with thrombotic antiphospholipid syndrome (APS). Lupus 2015;24:927-34.

16. Atsumi T, Furukawa S, Amengual O, Koike T. Antiphospholipid antibody associated thrombocytopenia and the paradoxical risk of thrombosis. Lupus 2005;14:499-504.

17. Demetrio Pablo R, Muñoz P, López-Hoyos M, Calvo V, Riancho L, Martínez-Taboada VM. Thrombocytopenia as a thrombotic risk factor in patients with antiphospholipid antibodies without disease criteria. Med Clin (Barc) 2017;148:394-400.

18. Abreu MM, Danowski A, Wahl DG, Amigo MC, Tektonidou M, Pacheco MS, et al. The relevance of "non-criteria" clinical manifestations of antiphospholipid syndrome: 14th International Congress on Antiphospholipid Antibodies Technical Task Force Report on Antiphospholipid Syndrome Clinical Features. Autoimmun Rev 2015; 14:401-14.

19. Proulle V, Furie RA, Merrill-Skoloff G, Furie BC, Furie $B$. Platelets are required for enhanced activation of the endothelium and fibrinogen in a mouse thrombosis model of APS. Blood 2014;124:611-22.

20. Comellas-Kirkerup L, Hernández-Molina G, Cabral AR. Antiphospholipid-associated thrombocytopenia or autoimmune hemolytic anemia in patients with or without definite primary antiphospholipid syndrome according to the Sapporo revised classification criteria: A 6-year follow-up study. Blood 2010;116:3058-63.

21. Toubi E, Shoenfeld Y. Livedo reticularis as a criterion for antiphospholipid syndrome. Clin Rev Allergy Immunol 2007;32:138-44.

22. Matyja-Bednarczyk A, Swadźba J, Iwaniec T, Sanak M, Dziedzina S, Ćmiel A, et al. Risk factors for arterial 
thrombosis in antiphospholipid syndrome. Thromb Res 2014;133:173-6.

23. Gamoudi D, Cutajar M, Gamoudi N, Camilleri DJ, Gatt A. Achieving a satisfactory clinical and biochemical response in antiphospholipid syndrome and severe thrombocytopenia with rituximab: Two case reports. Clin Case Rep 2017;5:845-8.

24. Pons-Estel GJ, Andreoli L, Scanzi F, Cervera R, Tincani A. The antiphospholipid syndrome in patients with systemic lupus erythematosus. J Autoimmun 2017;76:10-20.
25. Martirosyan A, Petrek M, Kishore A, Manukyan G. Immunomodulatory effects of therapeutic plasma exchange on monocytes in antiphospholipid syndrome. Exp Ther Med 2016;12:1189-95.

26. Ruiz-Irastorza G, Egurbide MV, Ugalde J, Aguirre C. High impact of antiphospholipid syndrome on irreversible organ damage and survival of patients with systemic lupus erythematosus. Arch Intern Med 2004;164:77-82. 